\title{
Evaluation of clinical outcomes of pancreatic cancer patients using circulating nucleic acids
}

\author{
Kunihiro Tsuchida \\ Division for Therapies Against Intractable Diseases, Institute for Comprehensive Medical Science (ICMS), Fujita Health University, Toyoake, Aichi, \\ Japan \\ Correspondence to: Kunihiro Tsuchida. Division for Therapies Against Intractable Diseases, Institute for Comprehensive Medical Science (ICMS), \\ Fujita Health University, Toyoake, Aichi 470-1192, Japan. Email: tsuchida@fujita-hu.ac.jp. \\ Provenance: This is an invited Editorial commissioned by Section Editor Dr. Rui Liao (Department of Hepatobiliary Surgery, The First Affiliated \\ Hospital of Chongqing Medical University, Chongqing, China). \\ Comment on: Bernard V, Kim DU, San Lucas FA, et al. Circulating Nucleic Acids Are Associated With Outcomes of Patients With Pancreatic Cancer. \\ Gastroenterology 2019;156:108-18.e4.
}

Received: 21 December 2018; Accepted: 26 December 2018; Published: 02 January 2019.

doi: $10.21037 / \operatorname{tgh} .2018 .12 .09$

View this article at: http://dx.doi.org/10.21037/tgh.2018.12.09

The incidence of pancreatic ductal adenocarcinomas (PDAC) is increasing, and, owing to its malignant features and poor prognosis, it is becoming the second or third leading cause of cancer death in many countries. Somatic mutations in KRAS, CDKN2A,TP53, and SMAD4 are frequently observed in PDAC (1). Bernard et al. examined circulating nucleic acid levels in liquid biopsies from 194 pancreatic cancer patients from April 2015 to October 2017 (2). Droplet quantitative digital polymerase chain reaction (ddPCR) was used to determine KRAS mutant allele fractions (MAFs) from circulating tumor cell DNA (ctDNA) and exosomal DNA (exoDNA) purified from a large series of prospectively collected plasma from patients. The authors showed that the baseline levels of CA19-9, a PDAC marker, and exoDNA and ctDNA affect the prognosis and overall survival of PDAC patients. In a longitude analysis, a MAF peak above $1 \%$ in exoDNA was significantly associated with radiological progression. This study indicated that longitude monitoring of exoDNA and ctDNA using liquid biopsies provides predictive and prognostic information for therapeutic intervention of pancreatic cancers with poor prognosis and metastasis.

In cancer diagnosis and clinical therapeutics, blood biomarkers are useful. In the case of PDAC, CA19-9 is frequently used. Recently, ctDNA in blood derived from tumors was found to be useful for the detection of various cancers including lung cancers, colorectal cancers, and PDAC (3-5). Early detection of genomic alterations by next generation sequencing in local tumors and ctDNA may be useful for determining optimal therapeutic strategies and predicting the prognosis of each patient (3-5). Exosomes, secreted small extracellular vesicles ranging from 40-150 nm in diameter with a lipid bilayer membrane, contain miRNA and mRNA from tumor tissues and circulate in plasma. They mediate transfer of nucleic acids between cells $(6,7)$. Double-stranded DNA is also found in exosomes and serves as a novel biomarker in cancer detection $(8,9)$. Genomic DNA from exosomes could be transferred from donor cells to recipient cells (10). Specific proteins are similarly encapsulated in exosomes, and are involved in signal transfer between cells (11). In the aforementioned study by Bernard et al., the authors compared the usefulness of tumor monitoring in localized and metastatic pancreatic tumors using paired exoDNA and ctDNA samples for diagnosis, prognosis, and therapeutic selection (2). One milliliter of plasma was used for ctDNA isolation, and $12 \mathrm{~mL}$ of plasma was used for exosome isolation. Exosomes were isolated by conventional sequential centrifugation. Patients with metastasis had a greater circulating MAF of KRAS. Although CA19-9 was able to predict disease progression without detectable exoKRAS mutant in some patients, a rise in KRAS MAF in exoDNA could be detected even before a rise of CA19-9 or CT imaging diagnosis in different patients. Therefore, these biomarkers should be used to complement each other.

ctDNA and exoDNA measurement in cancer patients 
are valuable for predicting overall survival and progressionfree survival beyond the detection and diagnosis of cancers. Combined measurement of ctDNA or exoDNA with CA19-9 and combination of ctDNA and exoKRAS MAF could predict survival. Therefore, multi-analyte approaches are useful not only for diagnosis but also for prognosis prediction and therapeutic selection for intractable PDAC. It is also of note that ctDNA is released from apoptotic or necrotic cells, whereas the majority of exoDNA is from viable cells.

Regarding exoDNA, purification of multiple samples by sequential centrifugation is not useful for high-throughput screening. Furthermore, although DNase-resistant intraexosomal DNAs are located within exosomes and protected by a phospholipid bilayer membrane $(9,10)$, several reports showed that exosomes carry DNAse I-sensitive nucleic acid on the surface $(12,13)$. Oncogenic genomic DNA either from inside or on the surface of exosomes could exhibit different levels of stability. In either case, sequential liquid biopsies to measure diagnostic and predictive nucleic acids such as ctDNA and exoDNA in PDAC patients have been found to be useful. Future studies using a tumor gene mutation panel to detect multiple mutated genes and ctDNA or exoDNA by liquid biopsies should be undertaken.

\section{Acknowledgements}

We thank H. Nikki March, PhD, from Edanz Group for proofreading.

\section{Footnote}

Conflicts of Interest: The author has no conflicts of interest to declare.

\section{References}

1. Takai E, Totoki Y, Nakamura H, et al. Clinical utility of circulating tumor DNA for molecular assessment in

doi: $10.21037 / \operatorname{tgh} .2018 .12 .09$

Cite this article as: Tsuchida K. Evaluation of clinical outcomes of pancreatic cancer patients using circulating nucleic acids. Transl Gastroenterol Hepatol 2019;4:2. pancreatic cancer. Sci Rep 2015;5:18425.

2. Bernard V, Kim DU, San Lucas FA, et al. Circulating Nucleic Acids Are Associated With Outcomes of Patients With Pancreatic Cancer. Gastroenterology 2019;156:108-18.e4.

3. Bettegowda C, Sausen M, Leary RJ, et al. Detection of circulating tumor DNA in early- and late-stage human malignancies. Sci Transl Med 2014;6:224ra24.

4. Pietrasz D, Pécuchet N, Garlan F, et al. Plasma Circulating Tumor DNA in Pancreatic Cancer Patients Is a Prognostic Marker. Clin Cancer Res 2017;23:116-23.

5. Sausen M, Phallen J, Adleff V, et al. Clinical implications of genomic alterations in the tumour and circulation of pancreatic cancer patients. Nat Commun 2015;6:7686.

6. Valadi H, Ekström K, Bossios A, et al. Exosome-mediated transfer of mRNAs and microRNAs is a novel mechanism of genetic exchange between cells. Nat Cell Biol 2007;9:654-9.

7. Lotvall J, Valadi H. Cell to cell signalling via exosomes through esRNA. Cell Adh Migr 2007;1:156-8.

8. Kahlert C, Melo SA, Protopopov A, et al. Identification of double-stranded genomic DNA spanning all chromosomes with mutated KRAS and p53 DNA in the serum exosomes of patients with pancreatic cancer. J Biol Chem 2014;289:3869-75.

9. Thakur BK, Zhang H, Becker A, et al. Double-stranded DNA in exosomes: a novel biomarker in cancer detection. Cell Res 2014;24:766-9.

10. Cai J, Han Y, Ren H, et al. Extracellular vesicle-mediated transfer of donor genomic DNA to recipient cells is a novel mechanism for genetic influence between cells. J Mol Cell Biol 2013;5:227-38.

11. Ageta H, Ageta-Ishihara N, Hitachi K, et al. UBL3 modification influences protein sorting to small extracellular vesicles. Nat Commun 2018;9:3936.

12. Németh A, Orgovan N, Sódar BW, et al. Antibioticinduced release of small extracellular vesicles (exosomes) with surface-associated DNA. Sci Rep 2017;7:8202.

13. Fischer S, Cornils K, Speiseder T, et al. Indication of Horizontal DNA Gene Transfer by Extracellular Vesicles. PLoS One 2016;11:e0163665. 